\title{
Atretic Cephalocele With Hypertrichosis
}

\author{
Jameson T. Loyal, MD; Emily Farrell, MD; Joseph C. Pierson, MD
}

\section{PRACTICE POINTS}

- Atretic cephalocele is a rare diagnosis occurring on the scalp as a nodule with an overlying hair tuft or alopecia with or without a hair collar.

- Imaging is of utmost importance when presented with a tuft of hair on the midline to rule out intracranial extension and associated abnormalities.

To the Editor:

A 2-week-old female infant presented to our dermatology clinic for evaluation of a $4.0 \times 4.5-\mathrm{cm}$ pink-red patch with a $1-\mathrm{cm}$ central nodule and an overlying tuft of hair on the midline occipital region (Figure). The patient was born at 39 weeks' gestation to nonconsanguineous parents via a normal spontaneous vaginal delivery and had an unremarkable prenatal course with no complications since birth. The red patch and tuft of hair were noted at birth, and the parents reported that the redness varied somewhat in size throughout the day and from day to day. An initial neurologic workup revealed no gross neurologic abnormalities. A head ultrasound revealed a soft-tissue hypervascular nodule that appeared separate from bony structures but showed evidence of a necklike extension from the nodule to the underlying soft tissues. The ultrasound could not definitively rule out intracranial extension; gross brain structures appeared normal. The initial differential diagnosis consisted of a congenital hemangioma (either a rapidly involuting or noninvoluting subtype), meningioma, or cephalocele.

Consultation with the pediatric neurosurgery service was sought, and magnetic resonance imaging of the head was performed, which demonstrated a cystic lesion within the subcutaneous soft tissue in the midline posterior scalp approximately $2 \mathrm{~cm}$ above the torcula. There also was a thin stalk extending from the cyst and going through an osseous defect within the occipital bone and attaching to the falx cerebri. There was no evidence of any venous communication with the cerebral sinus tracts or intraparenchymal extension. No intracranial abnormalities were noted. Given the radiographic evidence, a presumptive diagnosis of an atretic cephalocele was made with the plan for surgical repair.

The patient was re-evaluated at 3 and 4 months of age; there were no changes in the size or appearance of the lesion, and she continued to meet all developmental milestones. At 9 months of age the patient underwent uncomplicated neurosurgery to repair the cephalocele. Histopathologic examination of the resected lesion was consistent with an atretic cephalocele and showed positive staining for epithelial membrane antigen, which further confirmed a meningothelial origin; no glial elements were identified. The postoperative course was uncomplicated, and the patient was healing well at a follow-up examination 2 weeks after the procedure.

This case highlights the importance of an extensive workup when a patient presents with a midline lesion and hypertrichosis. The patient's red patch, excluding the hair tuft, was reminiscent of a vascular malformation or hemangioma precursor lesion given the hypervascularity, the history of the lesion being present since birth, the lack of neurologic symptomatology, and the history of meeting all developmental milestones. The differential diagnosis for this patient was extensive, as many neurologic conditions present with cutaneous findings. Having central nervous system (CNS) and cutaneous comorbidities coincide underscores their common neuroectodermal origin during embryogenesis. ${ }^{1,2}$

From the College of Medicine, University of Vermont, Burlington. Drs. Farrell and Pierson are from the Division of Dermatology, University of Vermont Medical Center.

The authors report no conflict of interest.

Correspondence: Jameson T. Loyal, MD, Given \#287, UVM College of Medicine, 89 Beaumont Ave, Burlington, VT 05405-0068

(Jameson.Loyal@gmail.com). 

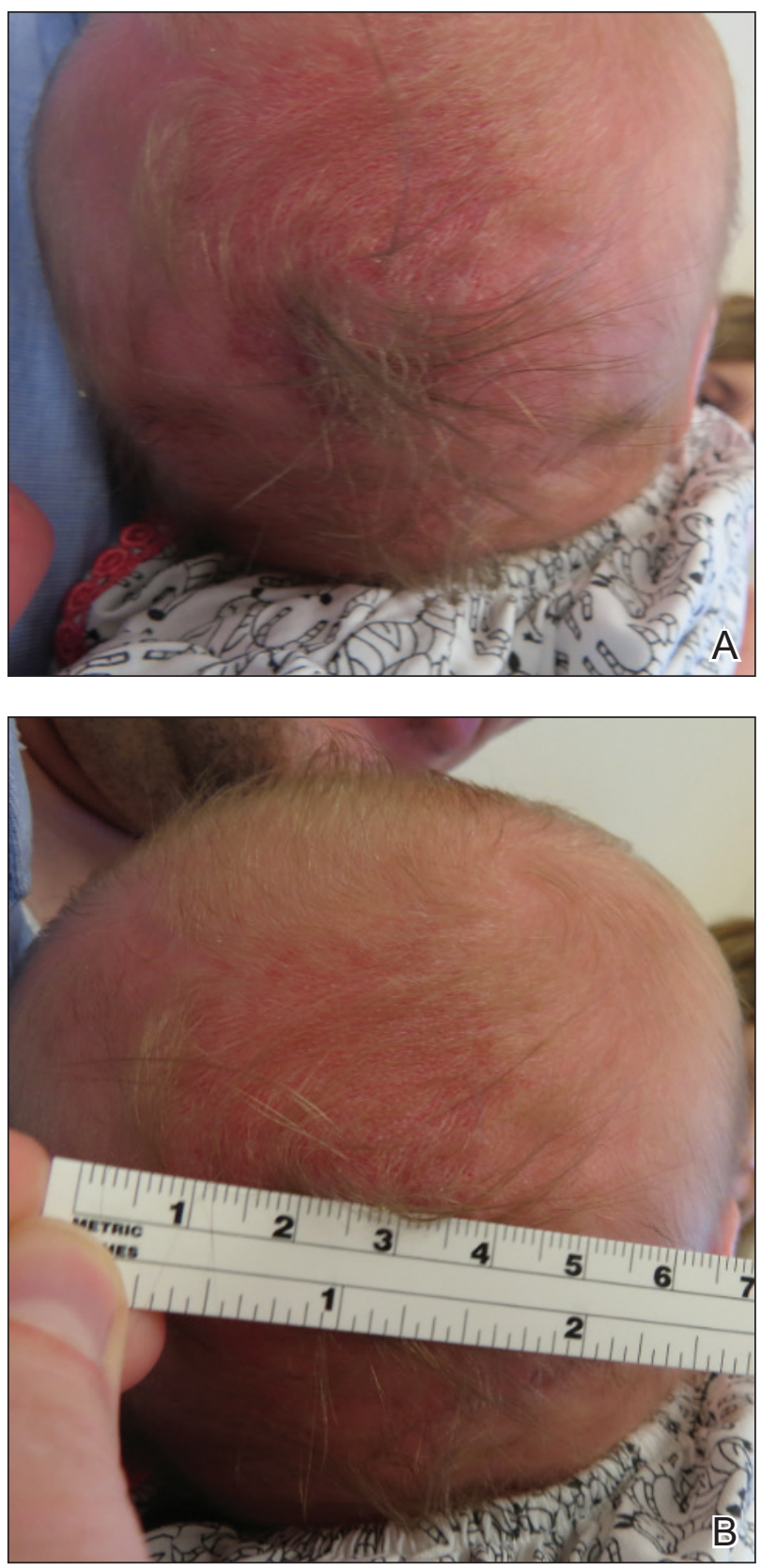

Atretic cephalocele. A, Overlying tuft of hair on the midline occipital region of the posterior scalp. B, A $4.0 \times 4.5-\mathrm{cm}$ pink-red patch with a 1 -cm central nodule and overlying tuft of hair.

Atretic cephalocele is a rare diagnosis, with the prevalence of cephaloceles estimated to be 0.8 to 3.0 per 10,000 births. $^{3}$ It typically occurs in either the parietal or occipital scalp as a skin nodule with a hair tuft or alopecic lesion with or without a hair collar. A cephalocele is defined as a skin-covered protrusion of intracranial contents through a bony defect. Central nervous system tissue, meninges, or cerebrospinal fluid can protrude outside the skull with this condition. An atretic cephalocele refers to a cephalocele that arrested in development and represents approximately $40 \%$ to $50 \%$ of all cephaloceles. ${ }^{4}$ Various hypotheses have explained the development of atretic cephaloceles: it represents a neural crest remnant, regression of a meningocele in utero, injury of multipotential mesenchymal cells, and either failure of the neural tube to close or reopening of the neural tube after closure. ${ }^{4-6}$ There is evidence of developmental defects in skin appendages including sweat and sebaceous glands, arrector pili muscles, and hair follicles in and around the skin overlying the cephalocele, suggesting that there is a developmental abnormality of not only the CNS but also the cutaneous tissue. ${ }^{5}$ Typical radiographic findings include a cystic lesion with underlying defect in the skull. A vertical positioning of the straight sinus also has been demonstrated to be a consistent finding that can aid in diagnosis. ${ }^{4}$

Imaging is of utmost importance when a patient presents with a tuft of hair on the scalp to rule out intracranial extension and associated abnormalities such as gray matter heterotopia, hypogenesis of the corpus callosum, hydrocephalus, and Dandy-Walker and Walker-Warburg syndromes, which have all been associated with atretic cephaloceles. ${ }^{4,7}$ The impact of location of the intracranial abnormality on prognosis has been contested, with some reporting a better prognosis with occipital cephalocele vs parietal cephalocele while others have found the opposite to be true. ${ }^{6,7}$ Surgical intervention typically is recommended to make a definitive diagnosis based on histologic evidence, to prevent ulceration or rupture of the lesion, and overall cosmesis. ${ }^{4,6}$

Cutaneous abnormalities presenting with hypertrichosis (ie, hair tuft, hair collar) and/or capillary malformations increase the likelihood of a cranial dysraphism, especially when these findings present together and occur in and around the midline. Clinical examination cannot rule out an underlying connection to the CNS; these findings require appropriate radiographic imaging assessment prior to any procedural intervention.

\section{REFERENCES}

1. Drolet BA, Clowry L, McTigue K, et al. The hair collar sign: marker for cranial dysraphism. Pediatrics. 1995;96(2, pt 1):309-313.

2. Sewell MJ, Chiu YE, Drolet BA. Neural tube dysraphism: review of cutaneous markers and imaging. Pediatr Dermatol. 2015;32:161-170.

3. Carvalho DR, Giuliani LR, Simão GN, et al. Autosomal dominant atretic cephalocele with phenotype variability: report of a Brazilian family with six affected in four generation. Am J Med Genet A. 2006;140:1458-1462.

4. Bick DS, Brockland JJ, Scott AR. A scalp lesion with intracranial extension. atretic cephalocele. JAMA Otolaryngol Head Neck Surg. 2015; 141:289-290.

5. Fukuyama M, Tanese K, Yasuda F, et al. Two cases of atretic cephalocele, and histological evaluation of skin appendages in the surrounding skin. Clin Exp Dermatol. 2016;41:48-52.

6. Martinez-Lage JF, Sola J, Casas C, et al. Atretic cephalocele: the tip of the iceberg. J Neurosurg. 1992;77:230-235.

7. Yakota A, Kajiwara H, Kohchi M, et al. Parietal cephalocele: clinical importance of its atretic form and associated malformation. J Neurosurg. 1988;69:545-551. 